\title{
Natural Gas Vehicle and Infrastructure Codes and Standards Citations
}

This document lists codes and standards typically used for U.S. natural gas vehicle and infrastructure projects. To determine which codes and standards apply to a specific project, identify the codes and standards currently in effect within the jurisdiction where the project will be located. Some jurisdictions also have unique ordinances or regulations that could apply.

Learn about codes and standards basics at www.afdc.energy.gov/afdc/codes_standards_basics.html.

Find natural gas vehicle and infrastructure codes and standards in these categories:

- $\quad$ Fire Code Requirements

- General CNG Requirements and Equipment Qualifications

- $\quad$ CNG Engine Fuel Systems

- CNG Compression, Gas Processing, Storage, and Dispensing Systems

- CNG Residential Fueling Facilities

- CNG Component Standards

\section{Fire Code Requirements}

\section{Approvals}

- International Fire Code 2208.2

\section{Location of dispensing operations and equipment}

- International Fire Code 2208.3

Private fueling of motor vehicles

- International Fire Code 2208.4

Pressure regulators

- International Fire Code 2208.5

\section{Valves}

- International Fire Code 2208.6

Emergency shutdown control

- International Fire Code 2208.7

Discharge of CNG from motor vehicle storage containers

- International Fire Code 2208.9

Containers, cylinders, and tanks (general)

- International Fire Code

Containers, cylinders, and tanks

- International Fire Code 3003.1 


\section{Design and construction}

- International Fire Code 3003.2

\section{Pressure relief devices}

- International Fire Code 3003.3

\section{Marking}

- International Fire Code 3003.4

\section{Security}

- International Fire Code 3003.5

Valve protection

- International Fire Code 3003.6

Separation from hazardous conditions

- International Fire Code 3003.7

Wiring and equipment

- International Fire Code 3003.8

Service and repair

- International Fire Code 3003.9

Unauthorized use

- International Fire Code 3003.1

Exposure to fire

- International Fire Code 3003.11

Leaks, damage, or corrosion

- International Fire Code 3003.12

Surface of unprotected storage or use areas

- International Fire Code 3003.13

Overhead cover

- International Fire Code 3003.14

\section{Lighting}

- International Fire Code 3003.15

\section{Vaults}

- International Fire Code 3003.16

\section{General CNG Requirements and Equipment Qualifications}

\section{Composition}

- $\quad$ NFPA 52 Gaseous Fuel Vehicular Systems Code 4.2

\section{System Approvals}

- NFPA 52 Gaseous Fuel Vehicular Systems Code 4.3

Design and Construction of Containers

- $\quad$ NFPA 52 Gaseous Fuel Vehicular Systems Code 4.4 


\section{Pressure Relief Devices}

- NFPA 52 Gaseous Fuel Vehicular Systems Code 4.5

\section{Pressure Gauges}

- NFPA 52 Gaseous Fuel Vehicular Systems Code 4.6

\section{Pressure Regulators}

- $\quad$ NFPA 52 Gaseous Fuel Vehicular Systems Code 4.7

\section{Fuel Lines}

- $\quad$ NFPA 52 Gaseous Fuel Vehicular Systems Code 4.8

\section{Valves}

- $\quad$ NFPA 52 Gaseous Fuel Vehicular Systems Code 4.9

\section{Hose and Hose Connections}

- NFPA 52 Gaseous Fuel Vehicular Systems Code 4.1

\section{Vehicle Fueling Connection}

- NFPA 52 Gaseous Fuel Vehicular Systems Code 4.11

\section{CNG Engine Fuel Systems}

\section{System Component Qualifications}

- NFPA 52 Gaseous Fuel Vehicular Systems Code 6.2 Installation of Fuel Supply Containers

- NFPA 52 Gaseous Fuel Vehicular Systems Code 6.3 Installation of Venting Systems

- NFPA 52 Gaseous Fuel Vehicular Systems Code 6.4

\section{Installation of Piping}

- NFPA 52 Gaseous Fuel Vehicular Systems Code 6.5

\section{Installation of Valves}

- NFPA 52 Gaseous Fuel Vehicular Systems Code 6.6

\section{Installation of Pressure Gauges}

- $\quad$ NFPA 52 Gaseous Fuel Vehicular Systems Code 6.7

Installation of Pressure Regulators

- NFPA 52 Gaseous Fuel Vehicular Systems Code 6.8

\section{Installation of Fueling Connection}

- NFPA 52 Gaseous Fuel Vehicular Systems Code 6.9

\section{Wiring Installation}

- NFPA 52 Gaseous Fuel Vehicular Systems Code 6.1

\section{Labeling}

- NFPA 52 Gaseous Fuel Vehicular Systems Code 6.11

\section{System Testing}

- NFPA 52 Gaseous Fuel Vehicular Systems Code 6.12 
System Maintenance and Repair

- NFPA 52 Gaseous Fuel Vehicular Systems Code 6.13

Discharge from Vehicle Containers

- NFPA 52 Gaseous Fuel Vehicular Systems Code 6.14

\section{CNG Compression, Gas Processing, Storage, and Dispensing Systems}

\section{System Component Qualifications}

- NFPA 52 Gaseous Fuel Vehicular Systems Code 8.2

\section{General System Requirements}

- $\quad$ NFPA 52 Gaseous Fuel Vehicular Systems Code 8.3

\section{System Siting}

- NFPA 52 Gaseous Fuel Vehicular Systems Code 8.4

Installation of Containers and Container Appurtenances (Other than Pressure Relief Devices)

- NFPA 52 Gaseous Fuel Vehicular Systems Code 8.5

Installation of Pressure Relief Devices

- NFPA 52 Gaseous Fuel Vehicular Systems Code 8.6

Installation of Pressure Regulators

- $\quad$ NFPA 52 Gaseous Fuel Vehicular Systems Code 8.7

Installation of Pressure Gauges

- $\quad$ NFPA 52 Gaseous Fuel Vehicular Systems Code 8.8

Installation of Piping and Hoses

- $\quad$ NFPA 52 Gaseous Fuel Vehicular Systems Code 8.9

\section{System Testing}

- NFPA 52 Gaseous Fuel Vehicular Systems Code 8.1

Installation of Emergency Shutdown Equipment

- NFPA 52 Gaseous Fuel Vehicular Systems Code 8.11

Installation of Electrical Equipment

- NFPA 52 Gaseous Fuel Vehicular Systems Code 8.12

Installation of Electrical Equipment

- NFPA 52 Gaseous Fuel Vehicular Systems Code 8.13

\section{System Operation}

- NFPA 52 Gaseous Fuel Vehicular Systems Code 8.14

\section{Fire Protection}

- NFPA 52 Gaseous Fuel Vehicular Systems Code 8.15

\section{System Maintenance}

- NFPA 52 Gaseous Fuel Vehicular Systems Code 8.16 
Vehicle Fueling Appliances in Nonresidential Occupancies

- $\quad$ NFPA 52 Gaseous Fuel Vehicular Systems Code 8.17

\section{CNG Residential Fueling Facilities}

- NFPA 52 Gaseous Fuel Vehicular Systems Code

\section{System Component Qualifications}

- NFPA 52 Gaseous Fuel Vehicular Systems Code 10.2

\section{General Safety Requirements}

- $\quad$ NFPA 52 Gaseous Fuel Vehicular Systems Code 10.3

\section{Installation}

- NFPA 52 Gaseous Fuel Vehicular Systems Code 10.4

\section{Installation of Pressure Relief Valves}

- NFPA 52 Gaseous Fuel Vehicular Systems Code 10.5

\section{Installation of Pressure Gauges}

- NFPA 52 Gaseous Fuel Vehicular Systems Code 10.6

\section{Pressure Regulation}

- $\quad$ NFPA 52 Gaseous Fuel Vehicular Systems Code 10.7

\section{Piping and Hose}

- $\quad$ NFPA 52 Gaseous Fuel Vehicular Systems Code 10.8

\section{Testing}

- NFPA 52 Gaseous Fuel Vehicular Systems Code 10.9

Installation of Emergency Shutdown Equipment

- NFPA 52 Gaseous Fuel Vehicular Systems Code 10.1

\section{Operation}

- NFPA 52 Gaseous Fuel Vehicular Systems Code 10.11

\section{Maintenance and Inspection}

- $\quad$ NFPA 52 Gaseous Fuel Vehicular Systems Code 10.12

\section{CNG Component Standards}

\section{CSA America}

- NGV 2 Natural Gas Vehicle Containers

- NGV 1 Compressed Natural Gas Vehicle (NGV) Fueling Connection Devices

- NGV 3.1Fuel System Components for Natural Gas Powered Vehicles

- NGV 4.1 Dispensing Systems

- NGV 4.2 Hoses for Natural Gas Vehicles and Dispensing Systems 
- NGV 4.3 Temperature Compensation Devices for Natural Gas Dispensing Systems

- NGV 4.4 Breakaway Devices for Natural Gas Dispensing Hoses and Systems

- NGV 4.5 (draft) Priority and Sequencing Equipment for Natural Gas Dispensing Systems

- NGV 4.6 Manually Operated Valves for Natural Gas Dispensing Systems

- NGV 4.7 (draft) Automatic Valves for Use in Natural Gas Vehicle Fueling Stations

- NGV 4.8 Natural Gas Fueling Station Reciprocating Compressor Guidelines

- PRD-1 Pressure Relief Devices for Natural Gas Vehicle (NGV) Fuel Container

\section{ASME}

- ASME B31.3 Process Piping

- Boiler and Pressure vessel Code

CGA

- C-6 Standards for Visual Inspection of Steel Compressed Gas Cylinders

- S-1.1 Pressure Relief Device Standards - Part 1- Cylinders for Compressed Gases

- S-1.3 Pressure Relief Device Standards - Part 3 - Stationary Storage Containers for Compressed Gases

SAE

- J1616 RP for Compressed Natural Gas Vehicle Fuel

- J 2406 RP for CNG Powered Medium and Heavy Duty Trucks 